\title{
Vibration Antiresonance Design for a Spacecraft Multifunctional Structure
}

\author{
Dong-Xu Li, Wang Liu, and Dong Hao \\ College of Aerospace Science and Engineering, National University of Defense Technology, Changsha, Hunan 410073, China \\ Correspondence should be addressed to Wang Liu; wangliu@nudt.edu.cn
}

Received 6 April 2017; Revised 7 July 2017; Accepted 19 July 2017; Published 27 August 2017

Academic Editor: Marc Thomas

Copyright (C) 2017 Dong-Xu Li et al. This is an open access article distributed under the Creative Commons Attribution License, which permits unrestricted use, distribution, and reproduction in any medium, provided the original work is properly cited.

Spacecraft must withstand rigorous mechanical environment experiences such as acceleration, noise, vibration, and shock during the process of launching, satellite-vehicle separation, and so on. In this paper, a new spacecraft multifunctional structure concept designed by us is introduced. The multifunctional structure has the functions of not only load bearing, but also vibration reduction, energy source, thermal control, and so on, and we adopt a series of viscoelastic parts as connections between substructures. Especially in this paper, a vibration antiresonance design method is proposed to realize the vibration reduction. The complex zero-point equations of the vibration system are firstly established, and then the vibration antiresonance design for the system is achieved. For solving the difficulties due to viscoelastic characteristics of the connecting parts, we present the determining formulas to obtain the structural parameters, so that the complex zero-point equations can be satisfied. Numerical simulation and ground experiment demonstrate the correctness and effectiveness of the proposed method. This method can solve the structural vibration control problem under the function constraints of load bearing and energy supplying and will expand the performance of spacecraft functional modules.

\section{Introduction}

Before getting into orbit and carrying out the normal work, spacecraft should undergo rigorous prelaunch environment. As we know, large level mechanical vibration connected with noise field and structural vibration will be generated during the launch process of rocket, and strong acceleration and severe noise will be accompanied during the ascending period of rocket. Furthermore, intensive mechanical shock will be caused by the satellite-vehicle separation and the operation of initiating explosive device and release mechanism, such as the deployment of solar arrays. Such vibration and shock loads will directly transfer to the spacecraft through the structure and hence bring a potential risk of damage of critical components or structures on spacecraft and accuracy reduction and functional degeneration of spacecraft system or payloads $[1,2]$. Therefore, adopting efficient structural vibration control skills to make sure the spacecraft can withstand the rigorous mechanical environment experiences, such as acceleration, noise, vibration, and shock in the boost phase of carrier rocket, is always an inevitable and close concerned problem in the field of aerospace engineering.

In recent two decades, with the development of multichip module technology, high performance composite material technology, flexible circuit connection technology, and advanced thermal control technology, the spacecraft multifunctional structure technology breaks away from cocoon and gets rapid development [3-6]. Such technology organically combines the functions of data processing, radiation protection, thermal control, electric power storage, and so on as a whole and achieves an integrated design of structure, function, and material, which can eliminate the redundant mass and volume of traditional satellite, improve the available free space and payload mass ratio of satellite, shorten the time period of satellite design and fabrication substantially, and extend the life of satellite effectively. Up to date, several researchers have developed such multifunctional structure technology, prompted a series of fundamental researches, and conducted spaceflight test verification on some key techniques. For example, NASA, Lockheed Martin Space 
Systems, and the U.S. Air Force Research Laboratory have performed a large number of studies on the multifunctional structure, which highly integrates structure, electron, thermal control, and radiation protection, and have produced such multifunctional structure.

In addition, some structural vibration control technologies such as particle damper, impact damper, particle tuned mass damper, and viscoelastic damper [7-10] are used in the aerospace field at present. A reaction wheel isolator assembly [11] made up of a set of six machined spring elements with bonded viscoelastic material was designed to reduce microvibration transmitted to the optical payload of Chandra Observatory. A $1 \mathrm{~Hz}$ isolator [12] based on constrained layer viscoelastic damping technology is used between the spacecraft bus and the optical payload for the James Webb Space Telescope. An active vibration isolation system [13], which consists of a piezoelectric stack actuator, is used to isolate sensitive spacecraft payloads from the spacecraft in satellite ultra-quiet isolation technology experiment. Particle impact dampers (PIDs) [14-16] are enclosures/containers or structural voids partially filled with one or more particles (e.g., small balls of metals, sand, ceramics, and glass), which work on by dissipating energy through collisions and frictions between the particles or between the particles and the wall of the enclosure. Such PIDs also have effective vibration absorption effect on the spacecraft.

In summary, the state of the art of spacecraft multifunctional structure technology has taken the functionalintegrations such as structure/battery, structure/electron, structure/cable, structure/thermal control, and structure/ protection into consideration. Most often the existing vibration control strategies are designed and applied to spacecraft in an independent way. However, the function integration of structure and vibration reduction and the functionalintegration design on structure have not been well considered as yet. In our research, we designed a new spacecraft multifunctional structure (MFS) concept, which incorporates the functions of load bearing, vibration reduction, energy source, thermal control, and so on. This paper briefly introduces such a multifunctional structure concept. In the design of such structure, we adopt a series of viscoelastic parts as connections between substructures. Considering the significance of structural vibration reduction for spacecraft or their payloads, a vibration antiresonance design method is especially proposed to realize the vibration reduction in the paper. This proposed method can give a new way to solve the structural vibration control problem under the function constraints of load bearing and energy supplying and will expand the performance of spacecraft functional modules.

The paper is organized as follows. Firstly, the structural composition and functions of the new-concept spacecraft multifunctional structure designed by us are described in brief, and its structural equivalent simplified model is established. Secondly, the complex zero-point equation of the vibration system is derived, and the principle of the vibration antiresonance design method is explained. Thirdly, for solving the difficulties due to viscoelastic characteristics of the connecting parts, the determining formulas of unknown structural parameters are given based on the obtained

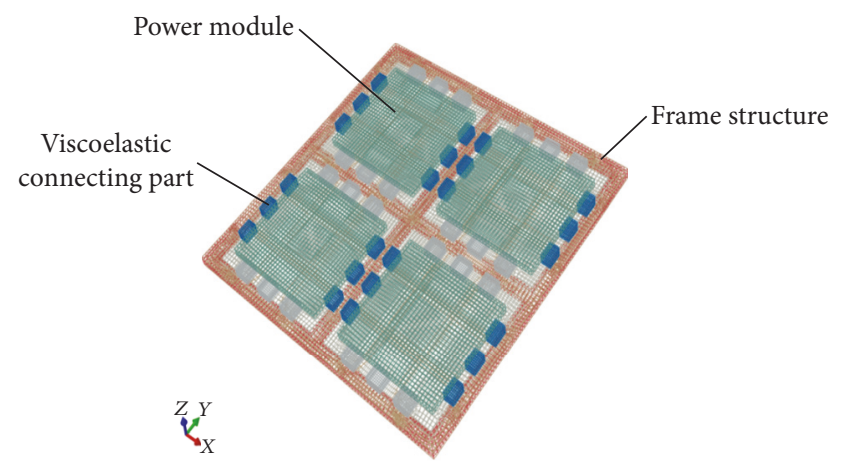

FIGURE 1: A spacecraft multifunctional structure combining load bearing, vibration reduction, and energy source.

complex zero-point equation, where the stiffness and damping parameters of the viscoelastic connecting parts are obtained by using the nonlinear mechanical constitutive relation model of the rubber materials. Finally, the effectiveness of the vibration control method proposed in this paper is verified by both the numerical simulation and ground experiment.

\section{System and Its Equivalent Simplified Model}

In order to improve the payload ratio of spacecraft and increase the available space in the spacecraft, we have proposed a new spacecraft MFS design concept. Such structure mainly consists of a frame structure, four power modules, and multiple viscoelastic connecting parts, as shown in Figure 1.

The frame structure, made of carbon fiber composite materials, is acting as the primary force bearing structure and has the function of load bearing. The power modules use allsolid-state polymer Li-ion batteries and have the functions of electric power supplying and storage. In addition, each power module can behave as an oscillator in a vibration control system to consume the system vibration energy. The viscoelastic connecting parts form a ring-shaped discontinuous cluster as shown in Figure 1. Each viscoelastic connecting parts member can provide an elastic structural support for the power modules and meanwhile provide a good vibration protection for the power modules by generating elastic deformation to dissipate part of the vibration energy. Furthermore, the viscoelastic connecting parts have the capacities of insulation and heat-sinking, since they are made from a kind of high polymer material that is known as silicone rubber.

From Figure 1, we can see that the global configuration of the frame structure is taken on a crisscross shape and thus the frame structure forms four squared cavities. In the center of each squared cavity, there exists a power module. The whole multifunctional structure is symmetric about the $x-, y$-, and $z$-axis. Here, coordinate system $o$-xyz denotes the rectangular inertial coordinate system of the multifunctional structure, where the $x$-axis and $y$-axis are perpendicular to each other and are in a plane parallel to the power module surface; the $z$-axis is perpendicular to $x o y$ plane and determined by the right-hand rule. The viscoelastic connecting parts are used 


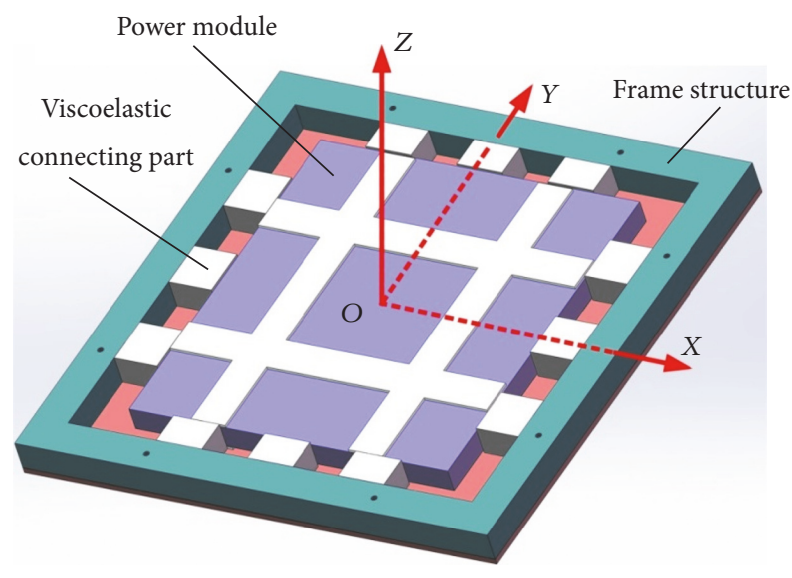

FIGURE 2: A quarter of the spacecraft multifunctional structure.

to connect the frame structure and the power modules at a precompressed way, and this connection is just surfacecontact, which means the viscoelastic connecting parts are not fixed or limited in determined positions.

By embedding the power modules into the interior of structure at an elastic restraint way, the spacecraft multifunctional structure achieves a highly combination of multifunction such as structural load bearing, electric power supplying, vibration reduction, and thermal control. At the same time, by applying a variety of lightweight materials such as carbon fiber composite materials in the fabrication of such multifunctional structure, the total structural mass is reduced drastically. Since the original space used for mounting the electric power systems is totally saved, the available space of spacecraft payloads is improved largely. Consequently, the multifunctional structure technology used in such structure has improved the payload mass ratio, the payload volume ratio, and the function structure ratio of the system platform significantly.

For convenience of subsequent discussions, we just choose a quarter of the structure model shown in Figure 1 as the analysis object according to the axial symmetry properties of the structure. The final-obtained simplified physical model is shown in Figure 2.

In Figure 2, because the stiffness of the power module is far greater than that of the viscoelastic connecting parts and thus the power module almost cause no elastic deformation in the vibration process, the power module could be equivalent as a seismic mass (denoted by $m_{2}$ ), which just has a mass property influence on the structural dynamic responses. The frame structure is the primary load bearing structure with high stiffness and high strength; thus, it can also be taken as a seismic mass (denoted by $m_{1}$ ). Since the viscoelastic connecting parts members are made from a high elasticity material and can be acting as the energy-sapping components that perform the function of vibration suppressing, they are equivalent as an assembly of a spring with certain stiffness and a damper with certain damping. The action effects of multiple viscoelastic connecting parts used for supporting the power

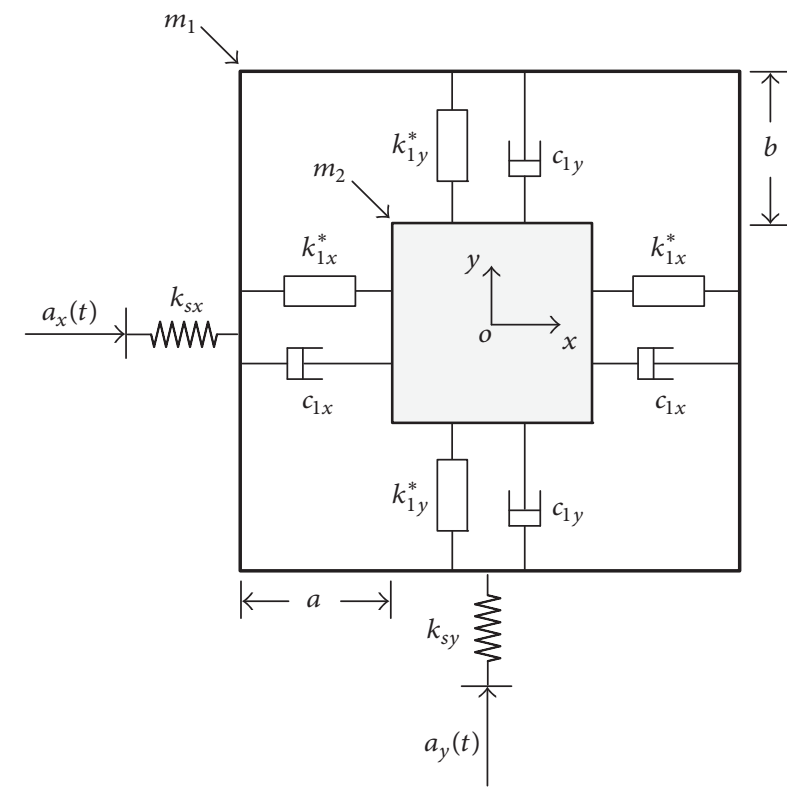

Figure 3: Equivalent vibration reduction model.

module are equal to that of connecting multiple springs and dampers in parallel. In this way, the equivalent model of the spacecraft multifunctional structure can be viewed as a constrained system with damping, as shown in Figure 3.

In Figure 3, $m_{1}$ and $m_{2}$ denote the mass of the frame structure and the power module, respectively; subscripts $x$ and $y$ denote the component of one physical variable in the $x$ direction and $y$ direction, respectively; $k_{1}^{*}$ and $c_{1}$ denote the complex stiffness and the damping of the viscoelastic connecting parts, respectively; $k_{s}$ denotes the structural stiffness of the spacecraft multifunctional structure; $a(t)$ denotes the external acceleration excitation, while $a$ and $b$ denote the distance between the edge of the power module and the frame structure in the $x$ and $y$ direction, respectively. According to the equivalent vibration reduction model, the frame structure, the power module, and the viscoelastic connecting parts constitute a vibration reduction system, which is the same as a spring-oscillator model, where the viscoelastic connecting parts are equal to elastic supports with certain stiffness and damping, and the power module is equal to an oscillator with certain mass. This vibration reduction system can partly absorb the vibration energy by the damping properties of viscoelastic connecting parts on the one hand and can partly consume the vibration energy by transferring the structural vibration energy to the kinetic energy of the oscillator on the other hand. The result of integration of the two factors above leads to a significant attenuation of vibration transmission in the structure and makes an efficient management for the mechanical environment of the power module.

\section{Complex Zero-Point Equation of System}

In this section, we discuss the detailed analysis of the equivalent simplified vibration reduction model shown in 


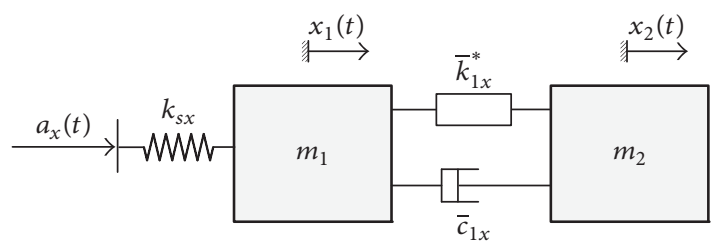

FIGURE 4: Equivalent simplified model in the $x$ direction.

Figure 3. Taking the axial symmetry of the model into consideration, and meanwhile supposing that the stiffness and damping provided by the spring and damper in the $y$ direction can be merged into those in the $x$ direction, we can thus obtain a more simplified system model in which the mechanical behaviors in the $x$ direction and in the $y$ direction are decoupled, as shown in Figure 4.

In Figure $4, \bar{k}_{1 x}^{*}$ and $\bar{c}_{1 x}$ denote the equivalent complex stiffness and damping in the $x$ direction, respectively. For this simplified model shown in Figure 4, the equations of motion of the vibration system can be written as

$$
\begin{aligned}
& m_{1} \ddot{x}_{1}+\bar{c}_{1 x}\left(\dot{x}_{1}-\dot{x}_{2}\right)+\bar{k}_{1 x}^{*}\left(x_{1}-x_{2}\right)+k_{s x} x_{1} \\
& =f_{1}\left(a_{x}\right), \\
& m_{2} \ddot{x}_{2}-\bar{c}_{1 x}\left(\dot{x}_{1}-\dot{x}_{2}\right)-\bar{k}_{1 x}^{*}\left(x_{1}-x_{2}\right)=0,
\end{aligned}
$$

where $f_{1}\left(a_{x}\right)$ is the external acceleration exciting force acting in the $x$ direction.

It can be further written in matrix form as

$$
[M]\{\ddot{x}\}+[C]\{\dot{x}\}+[K]\{x\}=\{f\},
$$

where $[M]=\left[\begin{array}{cc}m_{1} & 0 \\ 0 & m_{2}\end{array}\right]$ is the mass matrix, $[C]=\left[\begin{array}{cc}\bar{c}_{1 x} & -\bar{c}_{1 x} \\ -\bar{c}_{1 x} & \bar{c}_{1 x}\end{array}\right]$ is the damping matrix, $[K]=\left[\begin{array}{cc}\bar{k}_{1 x}^{*}+k_{s x} & -\bar{k}_{1 x}^{*} \\ -\bar{k}_{1 x}^{*} & \bar{k}_{1 x}^{*}\end{array}\right]$ is the stiffness matrix, $\{x\}=\left\{\begin{array}{l}x_{1} \\ x_{2}\end{array}\right\}$ denotes the input, and $\{f\}=\left\{\begin{array}{c}f_{1}\left(a_{x}\right) \\ 0\end{array}\right\}$ denotes the response.

Make the Fourier transformation for (2); we obtain

$$
\left([K]+j \omega[C]-\omega^{2}[M]\right)\{X(\omega)\}=\{F(\omega)\},
$$

where $j=\sqrt{-1}$.

In (3), the term of $[K]+j \omega[C]-\omega^{2}[M]$ has the property of stiffness, which hinders the movement of the system and can be called dynamic stiffness. Under the same excitation, the larger the dynamic stiffness is, the smaller the response will be. Hence, it is also known as impedance. For simplicity, we omit the subscript $x$ in the subsequent analysis: namely, we use $\bar{k}_{1}^{*}, \bar{c}_{1}$, and $k_{s}$ to substitute $\bar{k}_{1 x}^{*}, \bar{c}_{1 x}$, and $k_{s x}$, respectively. Thus, the impedance matrix of the system can be expressed as

$$
\begin{aligned}
& {[Z(\omega)]=[K]+j \omega[C]-\omega^{2}[M]} \\
& \quad=\left[\begin{array}{cc}
\bar{k}_{1}^{*}+k_{s}+j \omega \bar{c}_{1}-\omega^{2} m_{1} & -\bar{k}_{1}^{*}-j \omega \bar{c}_{1} \\
-\bar{k}_{1}^{*}-j \omega \bar{c}_{1} & \bar{k}_{1}^{*}+j \omega \bar{c}_{1}-\omega^{2} m_{2}
\end{array}\right] .
\end{aligned}
$$

The frequency response function of the system is the inverse matrix of the impedance matrix; namely,

$$
\begin{aligned}
& {[H(\omega)]=[Z(\omega)]^{-1}} \\
& =\frac{\left[\begin{array}{cc}
\bar{k}_{1}^{*}+k_{s}+j \omega \bar{c}_{1}-\omega^{2} m_{1} & \overline{1}_{1}^{*}+j \omega \bar{c}_{1} \\
\bar{k}_{1}^{*}+j \omega \bar{c}_{1} & \bar{k}_{1}^{*}+j \omega \bar{c}_{1}-\omega^{2} m_{2}
\end{array}\right]}{\left(\bar{k}_{1}^{*}+k_{s}+j \omega \bar{c}_{1}-\omega^{2} m_{1}\right)\left(\bar{k}_{1}^{*}+j \omega \bar{c}_{1}-\omega^{2} m_{2}\right)-\left(\bar{k}_{1}^{*}+j \omega \bar{c}_{1}\right)^{2}} .
\end{aligned}
$$

It further can be written as

$$
[H(\omega)]=\left[\begin{array}{lll}
H_{11}(\omega) & H_{12}(\omega) \\
H_{21}(\omega) & H_{22}(\omega)
\end{array}\right] .
$$
follows:

Therefore, the system equation can be expressed as

$$
\{X(\omega)\}=[Z(\omega)]^{-1}\{F(\omega)\}=[H(\omega)] \cdot\{F(\omega)\}
$$

or

$$
\left\{\begin{array}{l}
x_{1}(\omega) \\
x_{2}(\omega)
\end{array}\right\}=\left[\begin{array}{ll}
H_{11}(\omega) & H_{12}(\omega) \\
H_{21}(\omega) & H_{22}(\omega)
\end{array}\right]\left\{\begin{array}{c}
f_{1}(\omega) \\
0
\end{array}\right\} .
$$

In (6), each element $H_{l p}(\omega)$ in the frequency response function matrix has a specific physical meaning and plays a different role in the system transfer characteristics. Generally, $H_{l p}(\omega)$ is referred to as the frequency response function of point $l$ on the input of point $p$. When $l=p$, it is also known as the origin frequency response function; when $l \neq p$, it is called the cross-point frequency response function.

It can be obtained from ( 8 ) that

$$
x_{1}(\omega)=H_{11}(\omega) f_{1}(\omega) \text {, }
$$

where the origin frequency response function $H_{11}(\omega)$ is

$$
\begin{aligned}
H_{11}(\omega) & =\frac{\bar{k}_{1}^{*}+k_{s}+j \omega \bar{c}_{1}-\omega^{2} m_{1}}{\left(\bar{k}_{1}^{*}+k_{s}+j \omega \bar{c}_{1}-\omega^{2} m_{1}\right)\left(\bar{k}_{1}^{*}+j \omega \bar{c}_{1}-\omega^{2} m_{2}\right)-\left(\bar{k}_{1}^{*}+j \omega \bar{c}_{1}\right)^{2}} \\
& =\frac{\bar{k}_{1}^{*}+k_{s}+j \omega \bar{c}_{1}-\omega^{2} m_{1}}{\left(k_{s}-\omega^{2} m_{1}\right)\left(\bar{k}_{1}^{*}-\omega^{2} m_{2}\right)-\bar{k}_{1}^{*} \omega^{2} m_{2}+j \omega \bar{c}_{1}\left[k_{s}-\omega^{2}\left(m_{1}+m_{2}\right)\right]} .
\end{aligned}
$$




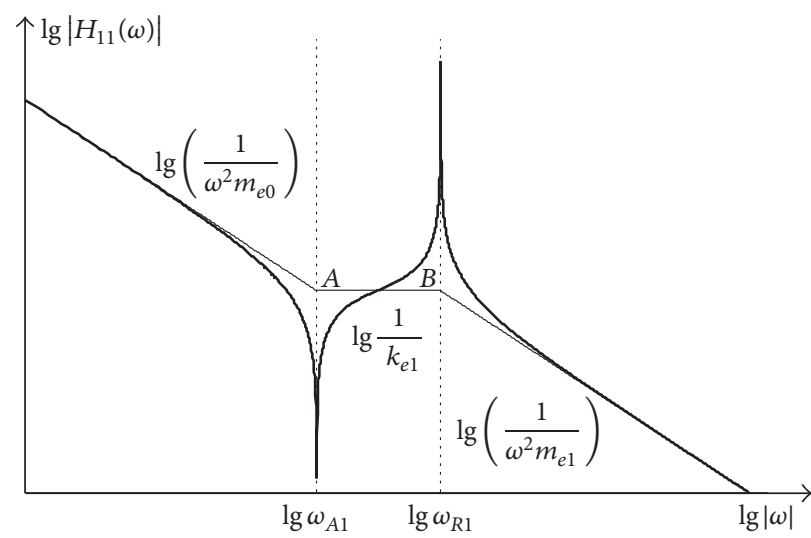

FIGURE 5: Log magnitude-frequency characteristic curve of system.

The origin frequency response function $H_{11}(\omega)$ is a complex number. Its real part and imaginary part can be, respectively, written as below

$$
\begin{aligned}
H_{11}^{R}(\omega) & =\frac{\left(\bar{k}_{1}^{*}+k_{s}-\omega^{2} m_{1}\right)\left[\left(k_{s}-\omega^{2} m_{1}\right)\left(\bar{k}_{1}^{*}-\omega^{2} m_{2}\right)-\bar{k}_{1}^{*} \omega^{2} m_{2}\right]+\omega^{2} \bar{c}_{1}^{2}\left[k_{s}-\omega^{2}\left(m_{1}+m_{2}\right)\right]}{\left[\left(k_{s}-\omega^{2} m_{1}\right)\left(\bar{k}_{1}^{*}-\omega^{2} m_{2}\right)-\bar{k}_{1}^{*} \omega^{2} m_{2}\right]^{2}+\omega^{2} \bar{c}_{1}^{2}\left[k_{s}-\omega^{2}\left(m_{1}+m_{2}\right)\right]^{2}}, \\
H_{11}^{I}(\omega) & =\frac{\omega \bar{c}_{1}\left[\left(k_{s}-\omega^{2} m_{1}\right)\left(\bar{k}_{1}^{*}-\omega^{2} m_{2}\right)-\bar{k}_{1}^{*} \omega^{2} m_{2}\right]-\omega \bar{c}_{1}\left[k_{s}-\omega^{2}\left(m_{1}+m_{2}\right)\right]\left(\bar{k}_{1}^{*}+k_{s}-\omega^{2} m_{1}\right)}{\left[\left(k_{s}-\omega^{2} m_{1}\right)\left(\bar{k}_{1}^{*}-\omega^{2} m_{2}\right)-\bar{k}_{1}^{*} \omega^{2} m_{2}\right]^{2}+\omega^{2} \bar{c}_{1}^{2}\left[k_{s}-\omega^{2}\left(m_{1}+m_{2}\right)\right]^{2}} \\
& =\frac{-\omega \bar{c}_{1}\left(k_{s}-\omega^{2} m_{1}\right)^{2}}{\left[\left(k_{s}-\omega^{2} m_{1}\right)\left(\bar{k}_{1}^{*}-\omega^{2} m_{2}\right)-\bar{k}_{1}^{*} \omega^{2} m_{2}\right]^{2}+\omega^{2} \bar{c}_{1}^{2}\left[k_{s}-\omega^{2}\left(m_{1}+m_{2}\right)\right]^{2}} .
\end{aligned}
$$

We have analyzed the amplitude-frequency characteristic of the origin frequency response function $H_{11}(\omega)$, to get its logarithmic amplitude-frequency characteristic curve, which is shown in Figure 5.

The following can be seen from Figure 5:

(1) When the denominator of $H_{11}(\omega)$ is equal to zero, namely, $\left|H_{11}(\omega)\right| \rightarrow \infty$, the system reaches a resonance state.

(2) When the numerator of $H_{11}(\omega)$ is equal to zero, $H_{11}(\omega)$ gets the minimum value $1 / k_{s}$. It can be known from
(9) that the vibration response (i.e., displacement $x_{1}$ ) of mass $m_{1}$ in the system becomes a minimum value, so the system is in the antiresonance state. The so-called antiresonance refers to the fact that, under the action of harmonic excitations with some specific frequencies, the elastic system causes the situation that the harmonic response of some parts of system is approximate to zero. In other words, the antiresonance situation means that the dynamic compliance of some parts of the system is approximate to zero at certain frequencies [1720]. Here, we called the equations

$$
\left|H_{11}(\omega)\right|=\left|\frac{\bar{k}_{1}^{*}+k_{s}+j \omega \bar{c}_{1}-\omega^{2} m_{1}}{\left(k_{s}-\omega^{2} m_{1}\right)\left(\bar{k}_{1}^{*}-\omega^{2} m_{2}\right)-\bar{k}_{1}^{*} \omega^{2} m_{2}+j \omega \bar{c}_{1}\left[k_{s}-\omega^{2}\left(m_{1}+m_{2}\right)\right]}\right|=0
$$




$$
\begin{aligned}
& H_{11}^{R}(\omega)=\frac{\left(\bar{k}_{1}^{*}+k_{s}-\omega^{2} m_{1}\right)\left[\left(k_{s}-\omega^{2} m_{1}\right)\left(\bar{k}_{1}^{*}-\omega^{2} m_{2}\right)-\bar{k}_{1}^{*} \omega^{2} m_{2}\right]+\omega^{2} \bar{c}_{1}^{2}\left[k_{s}-\omega^{2}\left(m_{1}+m_{2}\right)\right]}{\left[\left(k_{s}-\omega^{2} m_{1}\right)\left(\bar{k}_{1}^{*}-\omega^{2} m_{2}\right)-\bar{k}_{1}^{*} \omega^{2} m_{2}\right]^{2}+\omega^{2} \bar{c}_{1}^{2}\left[k_{s}-\omega^{2}\left(m_{1}+m_{2}\right)\right]^{2}}=0, \\
& H_{11}^{I}(\omega)=\frac{-\omega \bar{c}_{1}\left(k_{s}-\omega^{2} m_{1}\right)^{2}}{\left[\left(k_{s}-\omega^{2} m_{1}\right)\left(\bar{k}_{1}^{*}-\omega^{2} m_{2}\right)-\bar{k}_{1}^{*} \omega^{2} m_{2}\right]^{2}+\omega^{2} \bar{c}_{1}^{2}\left[k_{s}-\omega^{2}\left(m_{1}+m_{2}\right)\right]^{2}}=0
\end{aligned}
$$

as the basic complex zero-point equations of two-degreeof-freedom constrained system with damping, where superscripts $R$ and $I$ denote for the real part and imaginary part, respectively.

It is easy to derive that the conditions for making (14) hold are

$$
\begin{aligned}
& \varphi_{1}(\omega) \triangleq\left(\bar{k}_{1}^{*}+k_{s}-\omega^{2} m_{1}\right) \\
& \cdot\left[\left(k_{s}-\omega^{2} m_{1}\right)\left(\bar{k}_{1}^{*}-\omega^{2} m_{2}\right)-\bar{k}_{1}^{*} \omega^{2} m_{2}\right] \\
& +\omega^{2} \bar{c}_{1}^{2}\left[k_{s}-\omega^{2}\left(m_{1}+m_{2}\right)\right]=0, \\
& \varphi_{2}(\omega) \triangleq k_{s}-\omega^{2} m_{1}=0 .
\end{aligned}
$$

Antiresonance is an important parameter reflecting the local characteristics of vibration system (or the dynamic characteristics of excitation points), which is related to the physical parameters of some substructures in the system. The principle of vibration antiresonance design is that the structural parameters in the system should be reasonably designed to ensure that the complex zero-point equation of the vibration system will be satisfied: namely, the transfer function $H_{11}$ is satisfied to the minimum value condition; thus, the vibration of the concerned position in the system achieves a minimum level.

\section{Vibration Antiresonance Design}

For the spacecraft multifunctional structure described in this paper, the result that the frame structure will almost generate no vibration under external excitations is desired. According to the principle of antiresonance explained in the above section, we can choose appropriate parameters to make (13) or (14) hold, so that the vibration of the frame structure of the multifunctional structure remains at a minimum level under external exciting force $f_{1}$ and thus the purpose of vibration reduction is achieved. Supposing that the structural stiffness of the multifunctional structure $k_{s}$ and the mass of the frame structure $m_{1}$ are already known preliminarily, the main unknown parameters existing in the complex zero-point equation of the system mainly include the mass of the supported structure $m_{2}$, the stiffness of the viscoelastic connecting parts $\bar{k}_{1}^{*}$, and damping of the viscoelastic connecting parts $\bar{c}_{1}$. The following context will give a detailed discussion on the determining method of these parameters.
4.1. Stiffness and Damping of the Viscoelastic Connecting Parts. The viscoelastic connecting parts in the spacecraft multifunctional structure are an elastic member made of a rubber-like material, which has both the characteristics of an elastic solid and a viscous liquid under dynamic loadings. The elasticity demonstrates the stiffness characteristic of the member, while the viscous property demonstrates the damping characteristic of the member. Because the viscoelastic connecting parts have the anelasticity phenomenon, its stress-strain relationship is not linear. Therefore, it needs accurate viscoelastic constitutive model to predict its dynamic mechanical response. In this section, the Havriliak-Negami model (referred to as $\mathrm{H}$ $\mathrm{N}$ model) is used to model the constitutive properties of the viscoelastic connecting parts in spacecraft multifunctional structures.

By combining the complex modulus of the viscoelastic material $E^{*}$, with the low-frequency rubbery state modulus $E_{0}$ and the high-frequency glass state modulus $E_{\infty}$, the viscoelastic constitutive model based on the $\mathrm{H}-\mathrm{N}$ model is obtained as below [21]:

$$
E^{*}(\omega)=\frac{E_{0}-E_{\infty}}{\left[1+(i \omega \tau)^{\alpha}\right]^{\beta}}+E_{\infty}=E^{\prime}(\omega)+i E^{\prime \prime}(\omega),
$$

where $\omega$ is the angular frequency, $\tau$ is the relaxation time related to the temperature, and $i$ is the imaginary unit; $E_{0}, E_{\infty}$, $\alpha$, and $\beta$ are model parameters independent of temperature, and $0<\alpha, \beta<1, E^{\prime}(\omega)$ is the storage modulus of viscoelastic material, $E^{\prime \prime}(\omega)$ is the loss modulus of viscoelastic material. When $\omega \rightarrow 0, E^{*}=E_{0}$; when $\omega \rightarrow+\infty, E^{*}=E_{\infty}$.

The relationship curve between $E^{\prime}(\omega)$ and $E^{\prime \prime}(\omega)$ is commonly known as the Cole-Cole curve, as shown in Figure 6, where $O E^{\prime}$ denotes the storage modulus axis and $O E^{\prime \prime}$ denotes the loss modulus axis.

The parameters $E^{\prime}(\omega)$ and $E^{\prime \prime}(\omega)$ in the viscoelastic constitutive model based on $\mathrm{H}-\mathrm{N}$ model can be determined by an iterative method given in [21]. Thus, for the viscoelastic connecting parts structure with known shape, the computing formula of the stiffness coefficient $\bar{k}_{1}^{*}$ and the damping coefficient $\bar{c}_{1}$ can be given. In the paper, the viscoelastic connecting parts structure shown in Figure 2 is a solid cubic structure with length $L$, width $W$, and height $H$, respectively; thus, we have

$$
\begin{aligned}
& \bar{k}_{1}^{*}=\frac{n E^{\prime}(\omega) A}{L}=\frac{n E^{\prime}(\omega) W H}{L}, \\
& \bar{c}_{1}=\frac{n E^{\prime \prime}(\omega) A}{\omega L}=\frac{n E^{\prime \prime}(\omega) W H}{\omega L},
\end{aligned}
$$




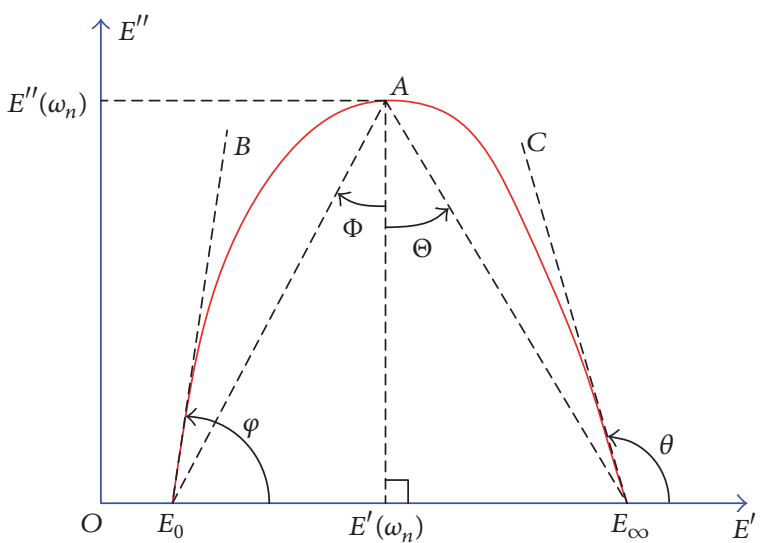

FIgure 6: Cole-Cole curve.

where $n$ is the number of viscoelastic connecting parts at one side of the power module.

It can be seen from (17) that, when the parameters of the viscoelastic constitutive model are determined based on the H-N model, the material storage modulus $E^{\prime}(\omega)$ and loss modulus $E^{\prime \prime}(\omega)$ of viscoelastic connecting parts can be obtained, and thus the stiffness coefficient $\bar{k}_{1}^{*}$ and damping coefficient $\bar{c}_{1}$ of viscoelastic connecting parts can be obtained.

4.2. Mass of the Power Module. According to the analysis result of Section 4.1, we think the stiffness and damping parameters of the viscoelastic connecting parts are already obtained by using the nonlinear mechanical constitutive relation model of the rubber materials. Based on the complex zero-point equation of the vibration system, the mass of the power module must be satisfied to the following equation:

$$
\begin{aligned}
& \left(\bar{k}_{1}^{*}+k_{s}-\omega^{2} m_{1}\right) \\
& \cdot\left[\left(k_{s}-\omega^{2} m_{1}\right)\left(\bar{k}_{1}^{*}-\omega^{2} m_{2}\right)-\bar{k}_{1}^{*} \omega^{2} m_{2}\right] \\
& +\omega^{2} \bar{c}_{1}^{2}\left[k_{s}-\omega^{2}\left(m_{1}+m_{2}\right)\right]=0,
\end{aligned}
$$

where $\omega$ is the vibration angular frequency of the system; we can use the antiresonance frequency need to be concerned in the calculation, which is the frequency under the antiresonance state.

By substituting (17) into (14), we can get the final complex zero-point equations of the spacecraft multifunctional structure with the viscoelastic material properties as follows:

$$
\begin{aligned}
& \left(\frac{n E^{\prime}(\omega) W H}{L}+k_{s}-\omega^{2} m_{1}\right) \\
& \cdot\left[\left(k_{s}-\omega^{2} m_{1}\right)\left(\frac{n E^{\prime}(\omega) W H}{L}-\omega^{2} m_{2}\right)\right. \\
& \left.-\frac{n E^{\prime}(\omega) W H}{L} \omega^{2} m_{2}\right]+\left(\frac{n E^{\prime \prime}(\omega) W H}{L}\right)^{2}\left[k_{s}\right. \\
& \left.-\omega^{2}\left(m_{1}+m_{2}\right)\right]=0,
\end{aligned}
$$

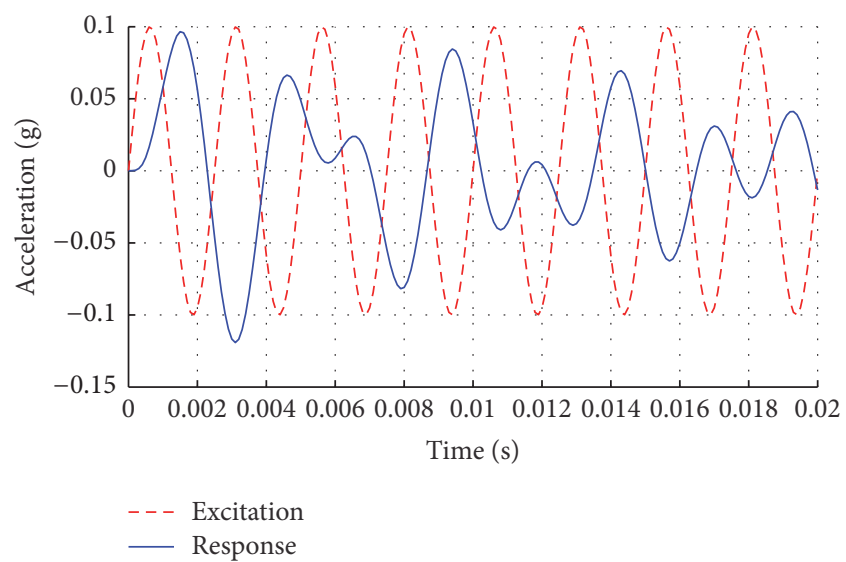

FIgURE 7: Acceleration response curve of the power module under harmonic excitation.

$$
\begin{aligned}
& \left(k_{s}-\omega^{2} m_{1}\right)\left(\frac{n E^{\prime}(\omega) W H}{L}-\omega^{2} m_{2}\right)-\frac{n E^{\prime}(\omega) W H}{L} \\
& \cdot \omega^{2} m_{2}-\left[k_{s}-\omega^{2}\left(m_{1}+m_{2}\right)\right]\left(\frac{n E^{\prime}(\omega) W H}{L}+k_{s}\right. \\
& \left.-\omega^{2} m_{1}\right)=0,
\end{aligned}
$$

where the physical dimensions including length $L$, width $W$, and height $H$ and the number $n$ of the viscoelastic connecting parts are the structural parameters need to be determined in the design of the spacecraft multifunctional structure.

\section{Verification of the Effectiveness of the Method}

In this section, both the numerical simulation and ground experiment are used to verify the effectiveness of the vibration antiresonance design method proposed in this paper.

5.1. Numerical Simulation Analysis. It is assumed that the frame structure of the spacecraft multifunctional structure is made of M40J composite material, whose Equivalent Young's modulus is $76.88 \mathrm{GPa}$, Poisson ratio is 0.319 , and mass density is $1510 \mathrm{~kg} / \mathrm{m}^{3}$. We suppose that the spacecraft multifunctional structure is under the action of a harmonic acceleration excitation. Here we analyze the structural response under harmonic excitation in the condition of $f=400 \mathrm{~Hz}$. The results of acceleration response of the power module and the frame structure in the $x$ direction are shown in Figure 7 , respectively.

We can know from Figure 7 that the response amplitude of the power module is less than that of the exciting loads, and the larger the frequency is, the smaller the response amplitude of the power module will be. Moreover, there is an obvious phase difference between the response amplitude of the power module and the exciting loads, and the higher the 


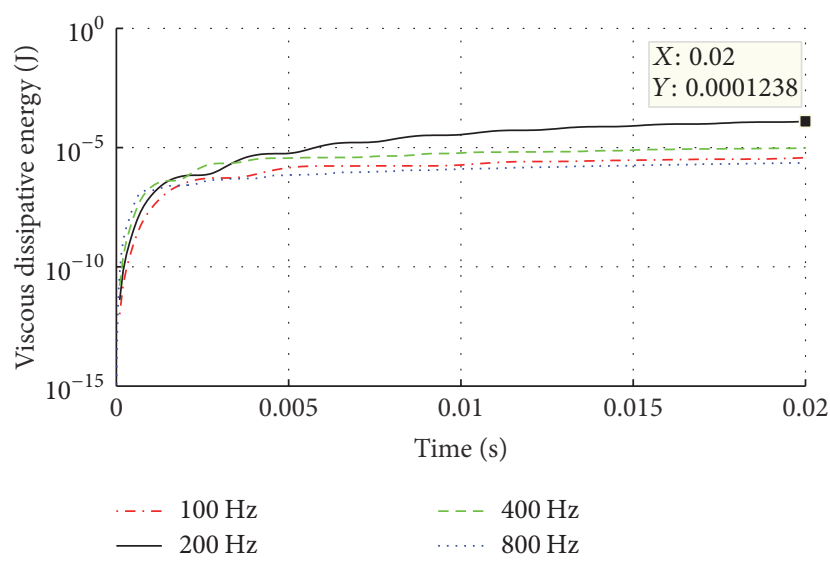

(a) The change of viscous dissipative energy with time

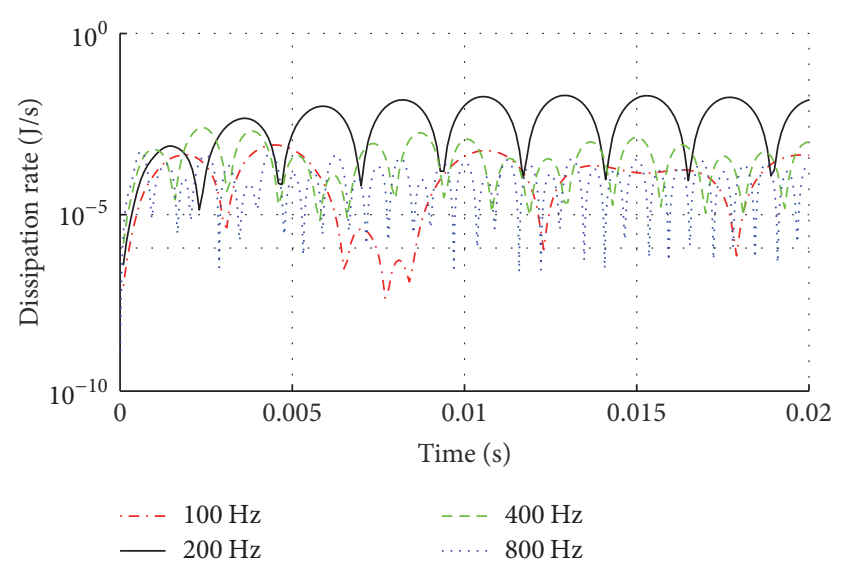

(b) The change of dissipation rate with time

FIGURE 8: Energy dissipation characteristics of spacecraft multifunctional structure under harmonic excitation.

exciting frequency, the more obvious the nonlinearity of the acceleration response of the power module.

Make a further analysis on the energy dissipation of the spacecraft multifunctional structure under harmonic excitation. Let the harmonic acceleration excitation be $a=$ $a_{0} \sin (\omega t)$, where $a_{0}=0.1 \mathrm{~g}\left(1 \mathrm{~g}=9800 \mathrm{~mm} / \mathrm{s}^{2}\right)$ and $\omega=2 \pi f$. We conduct a numerical simulation of the viscous dissipation energy and the dissipation rate on the conditions of $f=$ $100 \mathrm{~Hz}, f=200 \mathrm{~Hz}, f=400 \mathrm{~Hz}$, and $f=800 \mathrm{~Hz}$, and the time duration used in the simulation is set to be $0.02 \mathrm{~s}$. The viscous dissipation energy and the dissipation rate of the spacecraft multifunctional structure under harmonic excitation in the $\mathrm{x}$ direction are shown in Figures 8(a) and 8(b), respectively.

We can see from Figure 8 that the energy dissipation rate on the condition of $f=200 \mathrm{~Hz}$ (close to the resonance frequency of system) is much larger than that on the conditions of $f=100 \mathrm{~Hz}, f=400 \mathrm{~Hz}$, and $f=800 \mathrm{~Hz}$. When the exciting frequency is $f=200 \mathrm{~Hz}$, the viscous dissipation energy increases monotonically with time and reaches a maximum of $0.0001238 \mathrm{~J}$ (in the $\mathrm{x}$ direction) and $0.0001376 \mathrm{~J}$ (in the $\mathrm{y}$ direction) at the time of $0.02 \mathrm{~s}$. As to the viscous dissipation rate, it tends to change periodically with the increasing of time.

5.2. Ground Experimental Verification. The satellite is located in the fairing of rocket and will be subjected to the vibration load transmitted by the transition section during rocket launch. In order to analyze the dynamic characteristics of the spacecraft multifunctional structure under sinusoidal vibration loads, we conduct a sinusoidal vibration experiment research. The sinusoidal vibration experimental conditions are shown in Table 1, and the sinusoidal vibration experimental photo is shown in Figure 9.

In the dynamic test of the spacecraft multifunctional structure, the distribution of the acceleration pick-up sensors $\mathrm{A}, \mathrm{B}, \mathrm{C}$, and D is shown in Figure 10. The type of the acceleration pick-up sensors used in the experiment is BK4517 (Denmark Bruel \& Kjaer Ltd.), their physical sizes are $11.48 \mathrm{~mm} \times 6.35 \mathrm{~mm} \times 3.8 \mathrm{~mm}$, the sensitivity is $10 \mathrm{mV} / \mathrm{g}$,
TABLE 1: Sinusoidal vibration experimental conditions.

\begin{tabular}{lcc}
\hline Direction & Frequency $(\mathrm{Hz})$ & Level $\left(0-\mathrm{p}^{*}\right)$ \\
\hline \multirow{2}{*}{$x / y$} & $5 \mathrm{~Hz} \sim 17 \mathrm{~Hz}$ & $10.3 \mathrm{~mm}$ \\
& $17 \mathrm{~Hz} \sim 100 \mathrm{~Hz}$ & $12 \mathrm{~g}$ \\
\hline
\end{tabular}

*: "0-p" denotes single peak value.

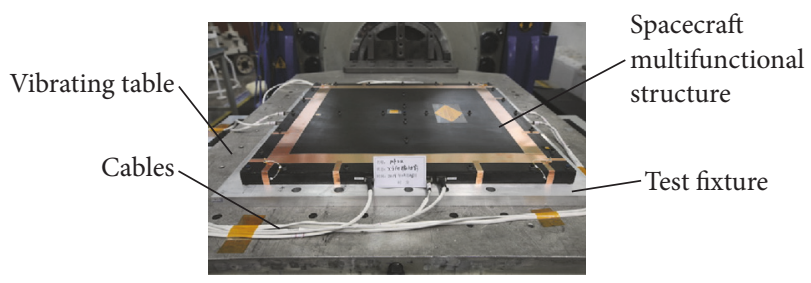

FIGURE 9: Live photo of the sinusoidal vibration experiment.

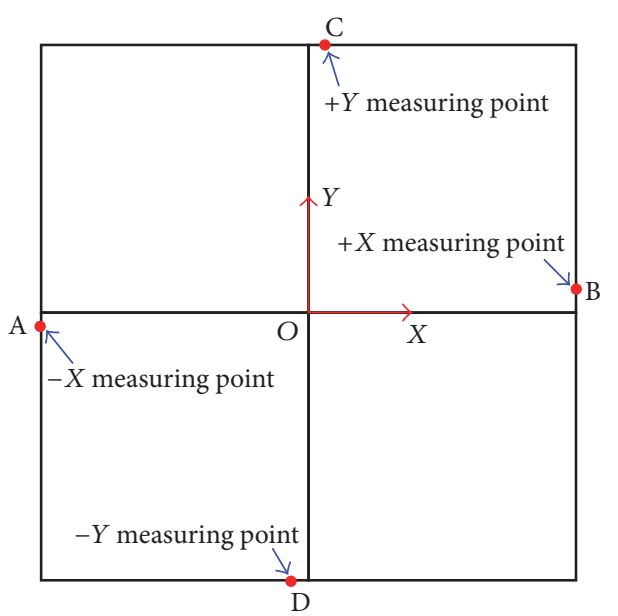

FIGURE 10: Distribution of acceleration measuring points.

the frequency range is from $1 \mathrm{~Hz}$ to $20000 \mathrm{~Hz}$, the resonance frequency is $55 \mathrm{kHz}$, the working temperature range is from $-55^{\circ} \mathrm{C}$ to $121^{\circ} \mathrm{C}$, and the mass is $0.6 \times 10^{-3} \mathrm{~kg}$. 


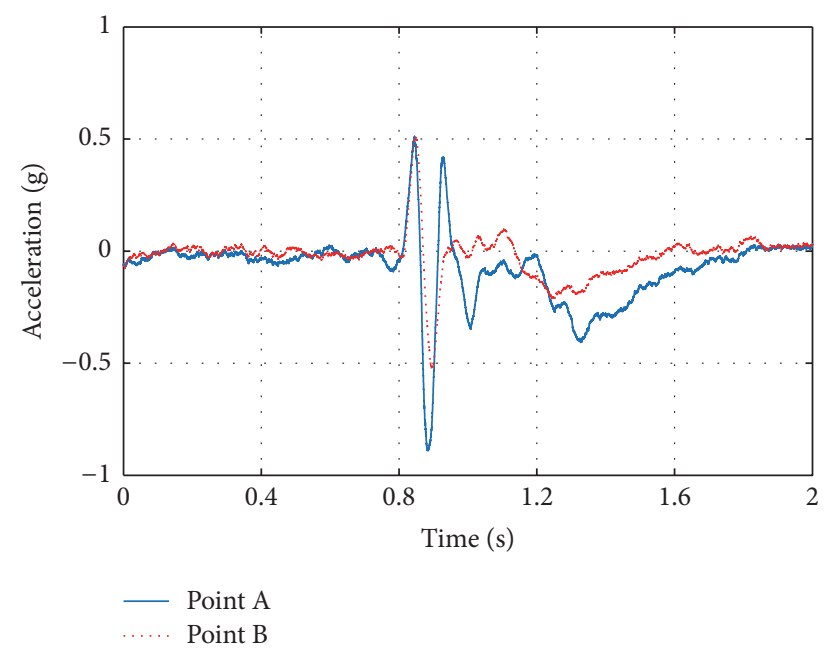

Figure 11: Sinusoidal vibration response spectrum in the $x$ direction.

The vibration response of the spacecraft multifunctional structure in the $x$ direction as measured by sensors $\mathrm{A}$ and $\mathrm{B}$ is shown in Figure 11.

From Figure 11, we can see that the acceleration output amplitude of measuring point $\mathrm{B}$ is obviously lower than that of measuring point $\mathrm{A}$. Hence, we can conclude that the external vibration is attenuated significantly through the transmission of the spacecraft multifunctional structure.

\section{Conclusions}

For the new spacecraft multifunctional structure design concept mainly composed of the frame structure, the power modules, and the viscoelastic connecting parts, a vibration antiresonance design method is proposed in this paper to reduce the vibration transmission through the structure. The basic complex zero-point equation of the vibration system is established. As to the special difficulties caused by the viscoelastic characteristics of the connecting parts, we deduce the determining formulas to obtain the structural parameters, such as the stiffness and damping of the viscoelastic connecting parts, which will make the complex zero-point equations hold. Furthermore, we get the final complex zeropoint equations of the spacecraft multifunctional structure, which has taken the viscoelastic material properties into account. Therefore, the vibration of the frame structure decreases under the action of external exciting forces and thus the wanted result of structural vibration reduction is achieved. Numerical simulation and ground experiment results show that the vibration reduction function of such spacecraft multifunctional structure is achieved successfully by using this vibration antiresonance design. The method proposed in this paper solves the structural vibration control problem under the function constraints of load bearing and energy supplying and expands the performance of spacecraft functional modules.

\section{Conflicts of Interest}

The authors declare that they have no conflicts of interest.

\section{References}

[1] P. Fortescue, G. Swinerd, and J. Stark, Spacecraft Systems Engineering, John Wiley \& Sons, 2011.

[2] T. Dunham, Aerospace Engineering: Design, Concepts and Applications, Clanrye International, 2016.

[3] S. C. Roberts and G. S. Aglietti, "Structural performance of a multifunctional spacecraft structure based on plastic lithiumion batteries," Acta Astronautica, vol. 67, no. 3-4, pp. 424-439, 2010.

[4] E. Harris N and R. Morgenthaler D, "Design \& testing of multifunctional structure concept for spacecraft," in Proceedings of the 41st AIAA/ASME/ASCE/AHS/ASC Structure, Structure Dynamics, and Materials Conference and Exhibit, Atlanta, Ga, USA, 2000.

[5] D. M. Barnett, S. Rawal, and K. Rummel, "Multifunctional structures for advanced spacecraft," Journal of Spacecraft and Rockets, vol. 38, no. 2, pp. 226-230, 2001.

[6] S. E. Hahn, R. Usami, and T. Ozaki, "Multifunctional structure spacecraft bus technology," in Proceedings of the A Collection of the 22nd AIAA International Communications Satellite Systems Conference and Exhibit, pp. 226-239, Monterey, Calif, USA, May 2004.

[7] Z. Xie, W. S. Shepard, and K. A. Woodbury, "Design optimization for vibration reduction of viscoelastic damped structures using genetic algorithms," Shock and Vibration, vol. 16, no. 5, pp. 455-466, 2009.

[8] X. P. Do, K. Shah, and S.-B. Choi, "Damping force tracking control of MR damper system using a new direct adaptive fuzzy controller," Shock and Vibration, vol. 2015, Article ID 947937, 2015.

[9] Z. Lu, D. Wang, and P. Li, "Comparison study of vibration control effects between suspended tuned mass damper and particle damper," Shock and Vibration, vol. 2014, Article ID 903780, 2014.

[10] Y. Du, S. Wang, Y. Zhu, L. Li, and G. Han, "Performance of a new fine particle impact damper," Advances in Acoustics and Vibration, vol. 2008, pp. 1-6, 2008.

[11] P. Y. Bely, K. J. Pendergast, C. J. Schauwecker, and J. B. Breckinridge, "Use of a passive reaction wheel jitter isolation system to meet the Advanced X-Ray Astrophysics Facility imaging performance requirements," in Proceedings of the Astronomical Telescopes \& Instrumentation, p. 1078, Kona, Hawaii, USA.

[12] A. J. Bronowicki, "Vibration isolator for large space telescopes," Journal of Spacecraft and Rockets, vol. 43, no. 1, pp. 45-53, 2006.

[13] V. Babuška, R. S. Erwin, and L. A. Sullivan, "System identification of the suite isolation platform: Comparison of ground and flight experiments," in Proceedings of the 44th AIAA/ASME/ASCE/AHS/ASC Structures, Structural Dynamics, and Materials Conference 2003, Norfolk, Va, USA, April 2003.

[14] V. L. Shinde and A. K. Pathak, "Review on particle damping technique for vibration suppression [J]," International Journal of Innovative Research in Science, Engineering and Technology, vol. 5, no. 3, pp. 2890-2895, 2016.

[15] V. U. Sakhare, A. S. Aradhye, and B. S. Gandhare, "A review on design and validation of performance of particle impact damper," International Journal of Current Engineering and Technology, vol. 6, no. 6, pp. 2038-2041, 2016. 
[16] P. S. Kachare and Bimleshkumar, "Effective location and packing ratio of a particle impact damper for a mild steel cantilever beam under transient and forced vibration," International Review of Mechanical Engineering, vol. 5, no. 7, pp. 1226-1234, 2011.

[17] E. Kausel, Advanced Structural Dynamics, Cambridge University Press, 2017.

[18] M. Berenguel and E. F. Camacho, "Frequency-based adaptive control of systems with antiresonance modes," Control Engineering Practice, vol. 4, no. 5, pp. 677-684, 1996.

[19] K. Jones and J. Turcotte, "Finite element model updating using antiresonant frequencies," Journal of Sound and Vibration, vol. 252, no. 4, pp. 717-727, 2002.

[20] V. Arora, "Use of resonance and antiresonance frequencies for better matching of frequency response function," International Journal of Structural Engineering, vol. 5, no. 1, pp. 13-23, 2014.

[21] D. Hao and D. Li, "Determination of dynamic mechanical properties of carbon black filled rubbers at wide frequency range using Havriliak-Negami model," European Journal of Mechanics, A/Solids, vol. 53, pp. 303-310, 2015. 


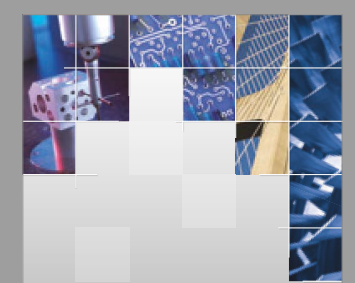

\section{Enfincering}
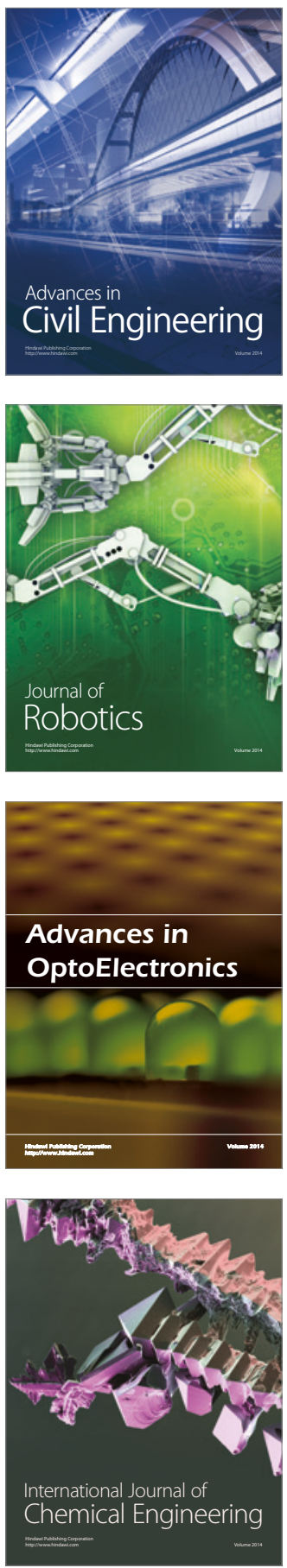

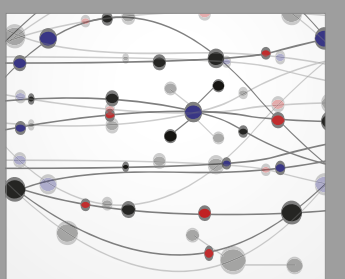

The Scientific World Journal

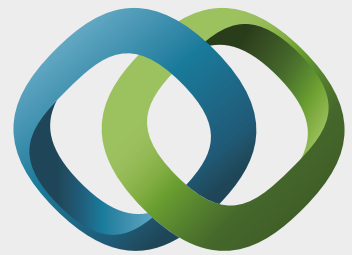

\section{Hindawi}

Submit your manuscripts at

https://www.hindawi.com
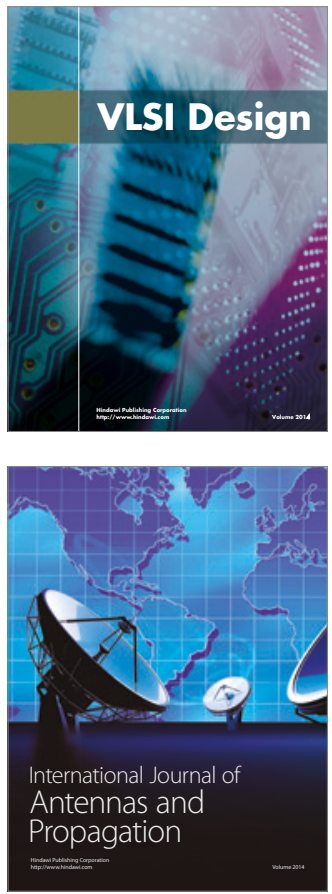

\section{Rotating}

Machinery
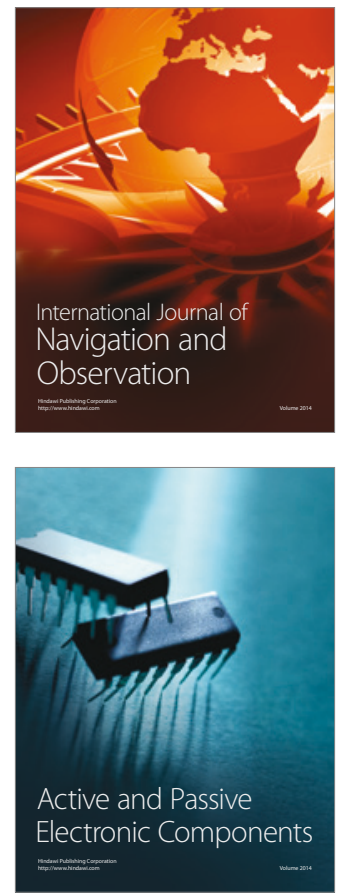
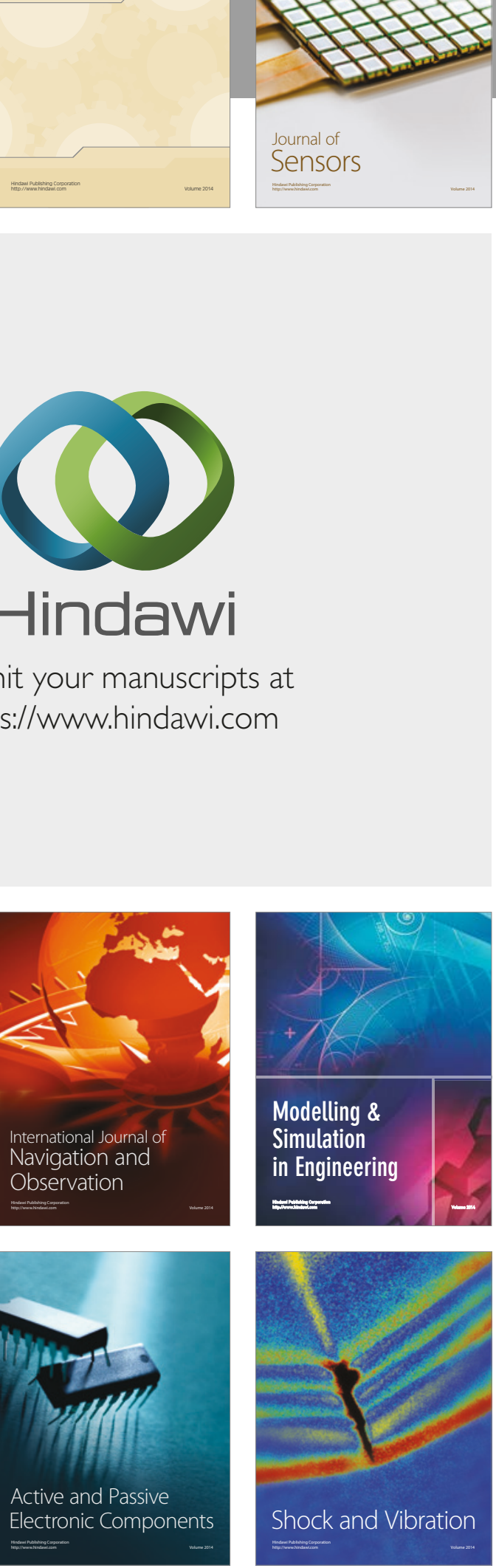
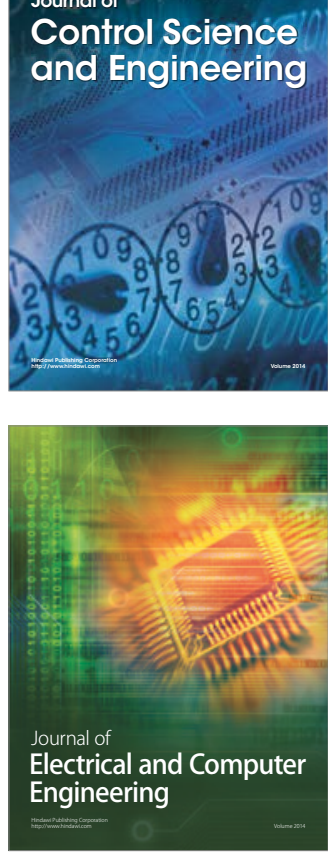

Distributed

Journal of

Control Science

and Engineering
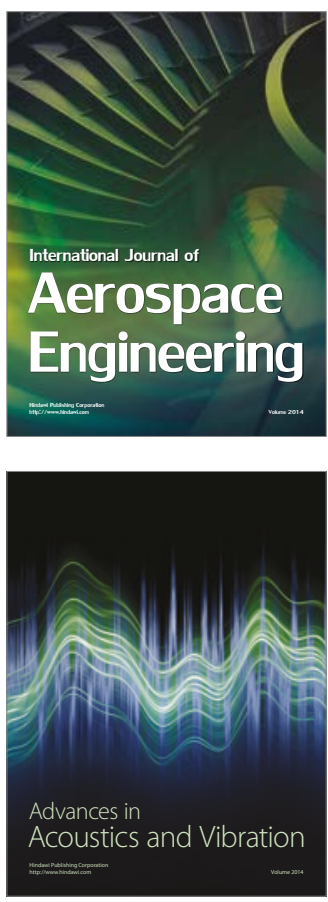

Sensor Networks 